\title{
The sub-Jupiter mass transiting exoplanet WASP-11b ${ }^{\star}$
}

\author{
R. G. West ${ }^{1}$, A. Collier Cameron ${ }^{2}$, L. Hebb ${ }^{2}$, Y. C. Joshi ${ }^{3}$, D. Pollacco ${ }^{3}$, E. Simpson ${ }^{3}$, I. Skillen ${ }^{4}$, H. C. Stempels ${ }^{2}$, \\ P. J. Wheatley ${ }^{5}$, D. Wilson ${ }^{6}$, D. Anderson ${ }^{6}$, S. Bentley ${ }^{6}$, F. Bouchy ${ }^{7,8}$, D. Christian ${ }^{3}$, B. Enoch ${ }^{9}$, N. Gibson ${ }^{2}$, \\ G. Hébrard ${ }^{7}$, C. Hellier ${ }^{6}$, B. Loeillet ${ }^{10}$, M. Mayor ${ }^{11}$, P. Maxted ${ }^{6}$, I. McDonald ${ }^{6}$, C. Moutou ${ }^{10}$, \\ F. Pont ${ }^{11}$, D. Queloz ${ }^{11}$, A. M. S. Smith ${ }^{2}$, B. Smalley ${ }^{6}$, R. A. Street ${ }^{12}$, and S. Udry ${ }^{11}$ \\ 1 Department of Physics \& Astronomy, University of Leicester, Leicester, LE1 7RH, UK \\ e-mail: rgw@astro.le.ac.uk \\ 2 School of Physics and Astronomy, University of St Andrews, North Haugh, St Andrews, Fife KY16 9SS, UK \\ 3 Astrophysics Research Centre, School of Mathematics \& Physics, Queen's University, University Road, Belfast, BT7 1NN, UK \\ 4 Isaac Newton Group of Telescopes, Apartado de Correos 321, 38700 Santa Cruz de la Palma, Tenerife, Spain \\ 5 Department of Physics, University of Warwick, Coventry CV4 7AL, UK \\ 6 Astrophysics Group, Keele University, Staffordshire, ST5 5BG, UK \\ 7 Institut d'Astrophysique de Paris, CNRS (UMR 7095) - Université Pierre \& Marie Curie, 98bis Bvd. Arago, 75014 Paris, France \\ 8 Observatoire de Haute-Provence, 04870 St Michel l'Observatoire, France \\ 9 Department of Physics and Astronomy, The Open University, Milton Keynes, MK7 6AA, UK \\ ${ }^{10}$ Laboratoire d'Astrophysique de Marseille, OAMP, Université Aix-Marseille \& CNRS, 38 rue Frédéric Joliot-Curie, \\ 13388 Marseille Cedex 13, France \\ 11 Observatoire de Genève, Université de Genève, 51 Ch. des Maillettes, 1290 Sauverny, Switzerland \\ 12 Las Cumbres Observatory, 6740 Cortona Dr. Suite 102, Santa Barbara, CA 93117, USA
}

Received 17 September 2008 / Accepted 20 May 2009

\section{ABSTRACT}

We report the discovery of a sub-Jupiter mass exoplanet transiting a magnitude $V=11.6$ host star 1SWASP J030928.54+304024.7. A simultaneous fit to the transit photometry and radial-velocity measurements yield a planet mass $M_{\mathrm{p}}=0.53 \pm 0.07 M_{\mathrm{J}}$, radius $R_{\mathrm{p}}=0.91_{-0.03}^{+0.06} R_{\mathrm{J}}$ and an orbital period of $3.722465_{-0.000008}^{+0.00006}$ days. The host star is an early to mid-K dwarf, with a spectral analysis yielding an effective temperature of $4800 \pm 100 \mathrm{~K}$ and $\log g=4.45 \pm 0.2$. It is amongst the smallest, least massive and lowest luminosity stars known to harbour a transiting exoplanet. WASP-11b is the third least strongly irradiated transiting exoplanet discovered to date, experiencing an incident flux $F_{\mathrm{p}}=1.9 \times 10^{8} \mathrm{erg} \mathrm{s}^{-1} \mathrm{~cm}^{-2}$ and having an equilibrium temperature $T_{\text {eql }}=960 \pm 70 \mathrm{~K}$.

Key words. stars: individual: WASP-11 - planetary systems - techniques: photometric - techniques: radial velocities techniques: spectroscopic

\section{Introduction}

Observations of planets that transit their host star represent the current best opportunity to test models of the internal structure of exoplanets and of their formation and evolution. Since the first detection of an exoplanetary transit signature (Charbonneau et al. 2000; Henry et al. 2000) over fifty transiting planetary systems have been identified. A number of wide-field surveys are in progress with the goal of detecting transiting exoplanets, for example OGLE (Udalski et al. 2002), XO (McCullough et al. 2005), HAT (Bakos et al. 2004), TrES (O’Donovan et al. 2006) and WASP (Pollacco et al. 2006).

The WASP project operates two identical instruments, at La Palma in the Northern hemisphere, and at Sutherland in South Africa in the Southern hemisphere. Each telescope has a field of view of just under 500 square degrees. The WASP survey is sensitive to planetary transit signatures in the light-curves of hosts in the magnitude range $V \sim 9-13$. A detailed description of the telescope hardware, observing strategy and pipeline data analysis is given in Pollacco et al. (2006).

* Photometric measurements and Table 2 are available in electronic form at the CDS via anonymous ftp to

cdsarc.u-strasbg.fr $(130.79 .128 .5)$ or via

http://cdsweb.u-strasbg.fr/cgi-bin/qcat?J/A+A/502/395
In this paper we report the discovery of WASP- $11 b^{1}$, a sub-Jupiter mass gas giant planet in orbit about the host star 1SWASP J030928.54+304024.7. We present the WASP discovery photometry plus higher precision optical follow-up and radial velocity measurements which taken together confirm the planetary nature of WASP-11b.

\section{Observations}

\subsection{WASP photometry}

The host star 1SWASP J030928.54+304024.7 (=USNOB1.0 1206-0039898 = 2MASS 03092855+3040249= TYC2 2340-1714-1 = NOMAD1 1206-0040974; hereafter labelled WASP-11) is catalogued as a star of magnitude $V=11.6$ with co-ordinates $\alpha=03^{\mathrm{h}} 09^{\mathrm{m}} 28^{\mathrm{s}} .541, \delta=+30^{\circ} 40^{\prime} 24^{\prime \prime} .85$ (J2000.0). WASP-11 was observed by SuperWASP-N during the 2004, 2006 and 2007 observing seasons, covering the intervals 2004 July 08 to 2004 September 29, 2006 September 09 to

\footnotetext{
1 It has become apparent that this object has been independently discovered by the NATnet project, with the HAT-10-P discovery paper submitted to ApJ 7 days after the submission of this paper. The two discovery groups have agreed on referring to it in future papers as WASP-11b/HAT-P-10b, with separate entries on www . exoplanet . eu
} 
Table 1. WASP-N survey coverage of WASP-11.

\begin{tabular}{cccccc}
\hline \hline Season & Camera & $N_{\text {pts }}$ & $N_{\text {tr }}$ & $\begin{array}{c}T_{0} \\
\text { BJD-2400000.0 }\end{array}$ & $\begin{array}{c}P \\
\text { (days) }\end{array}$ \\
\hline 2004 & 103 & 1756 & 4 & 53240.921696 & 3.7220 \\
2006 & 144 & 2679 & 3 & 54056.140758 & 3.7223 \\
2007 & 146 & 2750 & 2 & 54346.4883 & 3.7226 \\
2007 & 147 & 729 & 1 & - & - \\
\hline
\end{tabular}

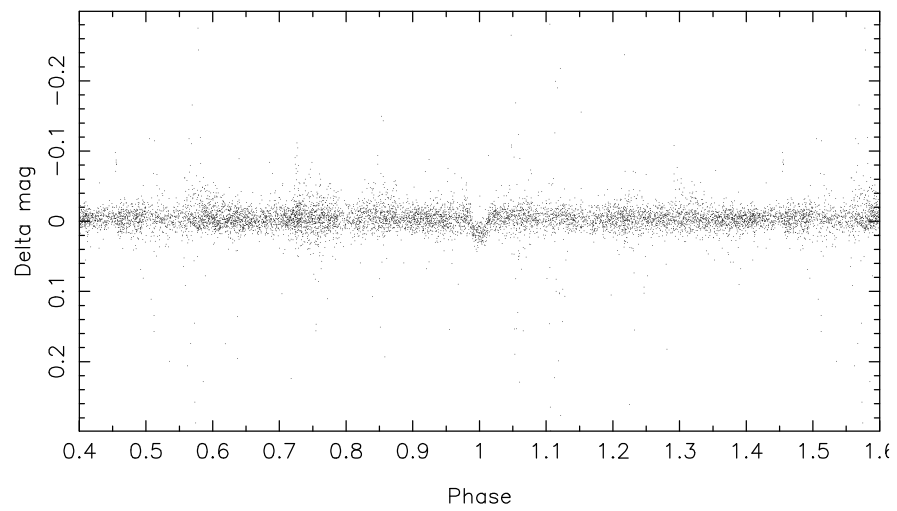

Fig. 1. SuperWASP-N photometry of WASP-11 from the 2004, 2006 and 2007 seasons. The data have been de-trended using the SYSREM scheme described in (Collier Cameron et al. 2006) and are plotted here phase-folded on the best-fit period from the MCMC analysis (Sect. 3).

2007 January 20 and 2007 September 04 to 2007 December 12 respectively. The pipeline-processed data were de-trended and searched for transits using the methods described in Collier Cameron et al. (2006), yielding a detection of a periodic transit-like signature with a period of 3.722 days. A total of ten transits are observed in data from all three observing seasons (Table 1, Fig. 1).

\subsection{Photometric follow-up}

WASP-11 was followed-up with the $2 \mathrm{~m}$ Liverpool telescope on La Palma as part of the Canarian Observatories' International Time Programme for $2007-08$. We used the $2048 \times 2048$ pixel EEV CCD42-40 imaging camera giving a scale of 0.27 arcsec/pixel in $2 \times 2$ bin mode and a total field of view of $\sim 4.6 \times 4.6^{\prime}$. Observations were taken during the transit of 2008 January 14, and consist of 656 images of 10 seconds exposure in the Sloan $z^{\prime}$ band. The night was non-photometric and with seeing varying from 0.9 to 2.2 arcsec during the four hour long observing run.

The images were bias subtracted and flat-field corrected with a stacked twilight flat-field image. All the science images were also corrected for the fringing effect. The autoguider did not work during our observations and a maximum positional shift of $17^{\prime \prime} .5$ of the stars within the frame was noticed. After aligning the images with respect to the first target image, aperture photometry were performed around the target and comparison stars using an aperture of 20 pixels (5'.4) radius. Three bright non-variable comparison stars were available in the target field with which to perform differential photometry. The resulting lightcurve from the Liverpool Telescope (Fig. 2 top panel) confirms the presence of a transit.

\subsection{Radial velocity follow-up}

Initial spectroscopic observations were obtained using the FIbrefed Echelle Spectrograph (FIES) mounted on the 2.5-m Nordic
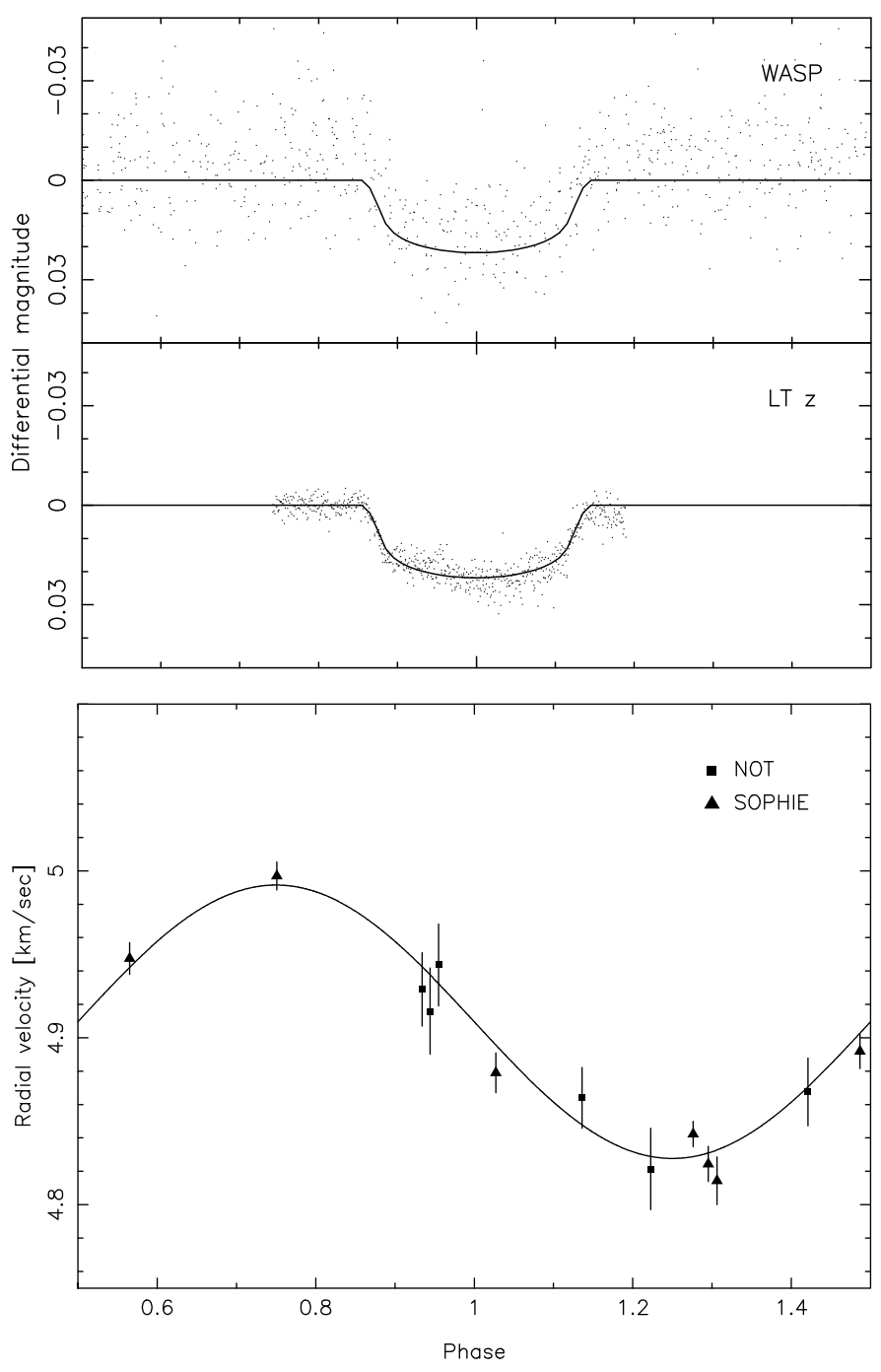

Fig. 2. The best-fit model from the simultaneous MCMC fit to the available photometry (top panel) and radial velocity data (lower panel). The fitted zero-point offset between the NOT and SOPHIE radial-velocity measurements $\left(5.4 \pm 0.4 \mathrm{~m} \mathrm{~s}^{-1}\right)$ is removed in this plot.

Optical Telescope. A total of 6 radial velocity points were obtained during 2007 December 27-31 and 2008 January 25. WASP-11 was observed with an exposure time of 1800s giving a signal-to-noise ratio of around $70-80$ at $5500 \AA$ A. FIES was used in medium resolution mode with $R=46000$ with simultaneous ThAr calibration. We used the bespoke data reduction package FIEStool to extract the spectra and a specially developed IDL line-fitting code to obtain radial velocities with a precision ${ }^{2}$ of $20-25 \mathrm{~m} \mathrm{~s}^{-1}$.

Radial velocity measurements of WASP-11 were also made with the Observatoire de Haute-Provence's 1.93 m telescope and the SOPHIE spectrograph (Bouchy \& The Sophie Team 2006), over the 5 nights 2008 February 11-15; a total of 7 usable spectra were acquired. SOPHIE is an environmentally stabilized spectrograph designed to give long-term stability at the level of a few $\mathrm{m} \mathrm{s}^{-1}$. We used the instrument in its medium resolution mode,

\footnotetext{
2 Stempels et al. (2007) report a radial velocity precision of $125 \mathrm{~m} \mathrm{~s}^{-1}$ in their analysis of FIES observations of WASP-1. The increased precision achieved in this paper is due primarily to the inclusion of simultaneous ThAr calibration spectra, which allows long-term instrumental drifts to be removed.
} 
Table 2. Radial velocity measurements of WASP-11.

\begin{tabular}{ccccc}
\hline \hline $\begin{array}{c}\text { BJD } \\
(\mathrm{UT})\end{array}$ & $\begin{array}{c}\mathrm{RV} \\
\left(\mathrm{km} \mathrm{s}^{-1}\right)\end{array}$ & $\begin{array}{c}\sigma_{R V} \\
\left(\mathrm{~km} \mathrm{~s}^{-1}\right)\end{array}$ & $v_{\text {span }}$ & Inst \\
\hline 2454462.395 & 4.8689 & 0.0185 & & NOT \\
2454463.456 & 4.8725 & 0.0203 & & NOT \\
2454465.404 & 4.9208 & 0.0258 & & NOT \\
2454466.440 & 4.8262 & 0.0244 & & NOT \\
2454466.443 & 4.9486 & 0.0246 & & NOT \\
2454491.424 & 4.9339 & 0.0220 & & NOT \\
2454508.3700 & 4.8910 & 0.0103 & 0.011 & SOPHIE \\
2454509.3534 & 5.0104 & 0.0084 & 0.000 & SOPHIE \\
2454510.3813 & 4.8989 & 0.0120 & 0.025 & SOPHIE \\
2454511.3092 & 4.8515 & 0.0076 & -0.002 & SOPHIE \\
2454511.3800 & 4.8330 & 0.0106 & -0.002 & SOPHIE \\
2454511.4206 & 4.8235 & 0.0143 & -0.035 & SOPHIE \\
2454512.3848 & 4.9482 & 0.0096 & 0.022 & SOPHIE \\
\hline
\end{tabular}

acquiring simultaneous star and sky spectra through separate fibres with a resolution of $R=48000$. Thorium-argon calibration images were taken at the start and end of each night, and at 2- to 3 -hourly intervals throughout the night. The radial-velocity drift never exceeded 2-3 $\mathrm{m} \mathrm{s}^{-1}$, even on a night-to-night basis.

Conditions during the SOPHIE observing run were photometric throughout, though all nights were affected by strong moonlight. Integrations of $1080 \mathrm{~s}$ yielded a peak signal-tonoise per resolution element of around $\sim 30-40$. The spectra were cross-correlated against a $\mathrm{K} 5 \mathrm{~V}$ template provided by the SOPHIE control and reduction software.

In all SOPHIE spectra the cross-correlation functions (CCF) were contaminated by the strong moonlight. We corrected them by using the CCF from the background light's spectrum (mostly the Moon) in the sky fibre, then scaled both CCFs using the difference of efficiency between the two fibres. Finally we subtracted the corresponding CCF of the background light from the star fibre, and fitted the resulting function by a Gaussian. The parameters obtained allow us to compute the photon-noise uncertainty of the corrected radial velocity measurement $\left(\sigma_{\mathrm{RV}}\right)$, using the relation

$\sigma_{\mathrm{RV}}=3.4 \sqrt{(F W H M)} /(S / N \times$ Contrast $)$.

Overall our SOPHIE RV measurements have an average photonnoise uncertainty of $10.3 \mathrm{~m} \mathrm{~s}^{-1}$. The measured barycentric radial velocity (Table 2, Fig. 2 lower panel) show a sinusoidal variation of half-amplitude $\sim 90 \mathrm{~m} \mathrm{~s}^{-1}$ about a centre-of-mass RV of $\sim 4.9 \mathrm{~km} \mathrm{~s}^{-1}$, consistent with the presence a companion of planetary mass. The period and ephemeris of the RV variation are consistent with those of found by the transit search.

An analysis of the line-bisector spans shows no significant correlation with radial velocity (Fig. 3), as would be expected if the observed radial velocity variations were due to a diluted eclipsing binary or chromospheric activity (Queloz et al. 2001).

\section{System parameters}

\subsection{Stellar parameters}

In order to perform a detailed spectroscopic analysis of the stellar atmospheric properties of WASP-11, we merged the available FIES spectra by first using the determined radial velocities to shift all spectra into the stellar rest frame, then using splineinterpolation to resample the spectra to a common wavelength grid and co-add the individual spectra into one high-quality spectrum. This merged spectrum was then continuum-normalized

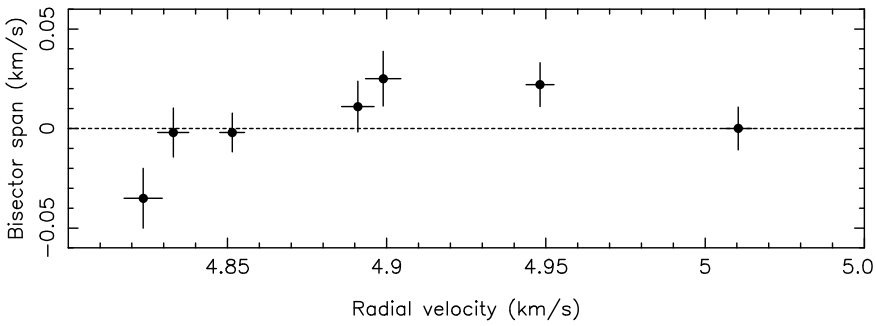

Fig. 3. The line bi-sector against velocity for WASP-11, showing no evidence of correlation.

Table 3. Coefficients of the limb-darkening model of Claret (2000) used in the MCMC fits to the photometry.

\begin{tabular}{lcccc}
\hline \hline Dataset & $a_{1}$ & $a_{2}$ & $a_{3}$ & $a_{4}$ \\
\hline WASP & 0.7265 & -0.7958 & 1.5198 & -0.6284 \\
LT & 0.7740 & -0.8258 & 1.2850 & -0.5339 \\
\hline
\end{tabular}

with a very low order polynomial to retain the shape of the broadest spectral features. The total signal-to-noise of the combined spectrum was around 200 per resolution element. We were not able to include the SOPHIE spectra in this analysis, because these spectra were obtained with the HE (high-efficiency) mode which is known to suffer from problems with removal of the blaze function.

For our analysis we followed the same procedure as for the spectroscopic characterization of WASP-1 (Stempels et al. 2007) and WASP-3 (Pollacco et al. 2008), we refer the reader to those papers for a detailed explanation of the spectral synthesis. A comparison between the observed FIES spectrum and the synthetic spectrum is shown in Fig. 4. The resulting fit parameters (Table 5) correspond to an early- to mid-K dwarf. A close examination of the region around the $\mathrm{Li}$ I 6708 line shows no evidence of such a feature, suggesting that the lithium abundance is very low.

\subsection{Planet parameters}

To determine the planetary and orbital parameters the SOPHIE and NOT FIES radial velocity measurements were combined with the photometry from WASP and the Liverpool Telescope in a simultaneous fit using the Markov Chain Monte Carlo (MCMC) technique. The details of this process are described in Collier Cameron et al. (2007) and Pollacco et al. (2008). As we were combining the radial velocity measurements from two telescopes we fitted a zero-point offset between the two datasets. The SOPHIE data was taken as the fiducial set, we fit a zeropoint offset for the NOT data of $5.4 \pm 0.4 \mathrm{~m} \mathrm{~s}^{-1}$. An initial fit showed that the orbital eccentricity $\left(e=0.086_{-0.062}^{+0.070}\right)$ was poorly constrained by the available data and nearly consistent with zero. We therefore fixed the eccentricity parameter at zero in a further fits. The limb-darkening of the host star was modelled using a linear interpolation of the models of Claret (2000), the coefficients used are listed in Table 3. Figure 2 shows the best-fitting models. The best-fit parameters (Table 4) assuming a circular orbit show WASP-11b to have a mass $M=0.53 \pm 0.07 M_{\mathrm{J}}$ and a radius of $R=0.91_{-0.03}^{+0.06} R_{\mathrm{J}}^{3}$.

\footnotetext{
${ }^{3}$ Planetary radii are here quoted in units of the equatorial radius of Jupiter, $R_{\mathrm{J}}=71492 \mathrm{~km}$.
} 

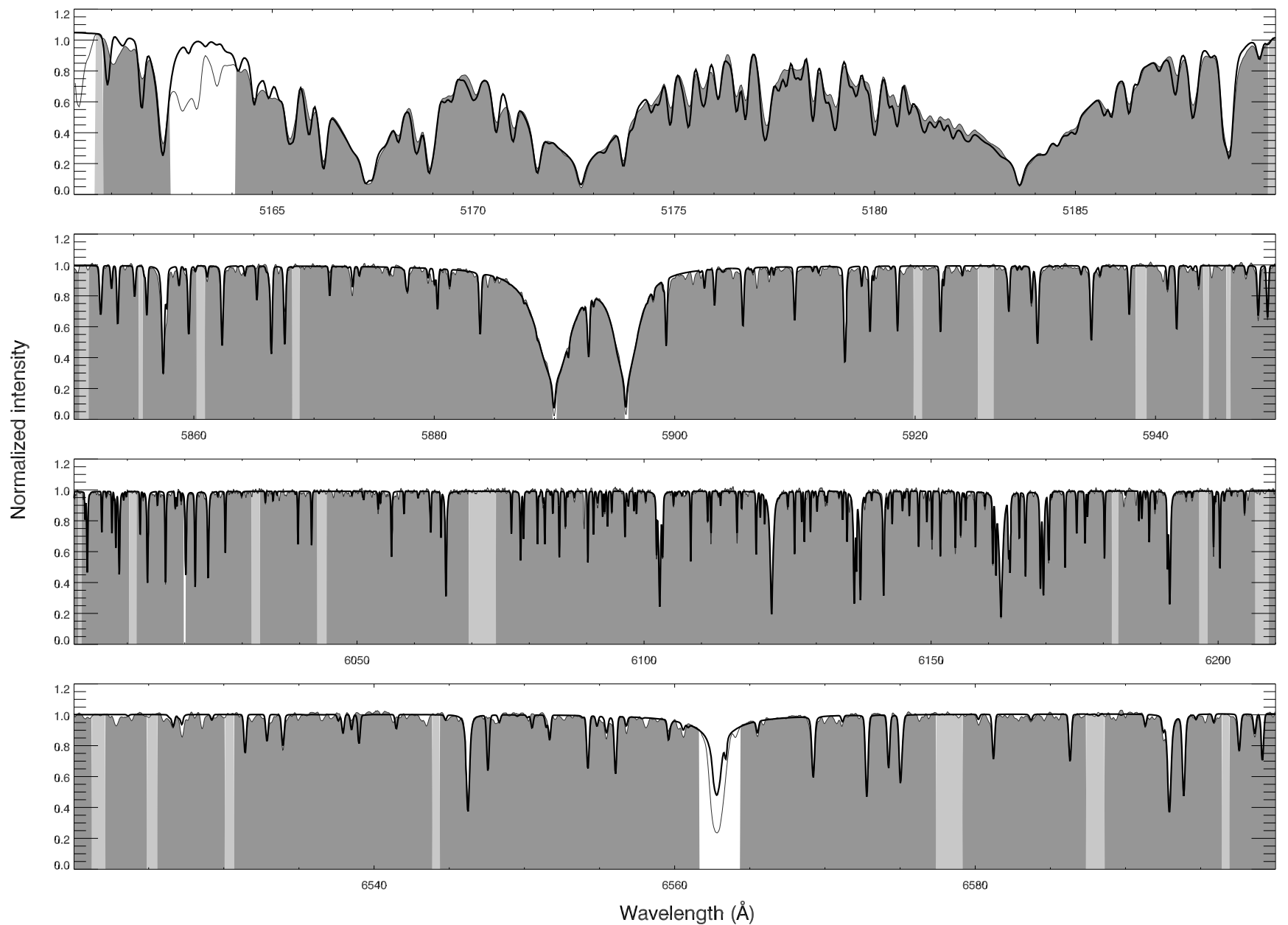

Fig. 4. A comparison between the observed FIES spectrum of WASP-11 and the calculated spectrum obtained from spectral synthesis with SME. The white regions are excluded from the spectral analysis, mainly because of the presence of telluric absorption. Light shaded regions were used to determine the continuum level, and the remaining dark shaded regions to determine the stellar atmospheric parameters.

Table 4. System parameters of WASP-11 derived from a simultaneous MCMC analysis of the available photometric and radialvelocity measurements. Quoted uncertainties define the $1 \sigma$ confidence intervals.

\begin{tabular}{lcc}
\hline \hline Parameter & Value & Unit \\
\hline Transit epoch (BJD), $T_{0}$ & $2454473.05588 \pm 0.00020$ & \\
Orbital period, $P$ & $3.722465_{-0.000008}^{+0.00006}$ & days \\
$\left(R_{\mathrm{p}} / R_{\star}\right)^{2}$ & $0.0162_{-0.0003}^{+0.0003}$ & \\
Transit duration & $2.556_{-0.007}^{+0.029}$ & hours \\
Impact parameter, $b$ & $0.054_{-0.050}^{+0.168}$ & $R_{*}$ \\
Reflex velocity, $K_{1}$ & $0.0821 \pm 0.0074$ & $\mathrm{~km} \mathrm{~s}^{-1}$ \\
Centre-of-mass velocity, $\gamma$ & $4.9077 \pm 0.0015$ & $\mathrm{~km} \mathrm{~s}^{-1}$ \\
Orbital eccentricity, $e$ & $\equiv 0.0($ adopted $)$ & \\
Orbital inclination, $i$ & $89.8_{-0.8}^{+0.2}$ & $\mathrm{deg}$ \\
Orbital separation, $a$ & $0.043 \pm 0.002$ & $\mathrm{AU}$ \\
Planet radius, $R_{\mathrm{p}}$ & $0.91_{-0.03}^{+0.06}$ & $R_{\mathrm{J}}$ \\
Planet mass, $M_{\mathrm{p}}$ & $0.53 \pm 0.07$ & $M_{\mathrm{J}}$ \\
log $g_{\mathrm{p}}$ (cgs) & $3.16_{-0.05}^{+0.04}$ & \\
Planet density, $\rho_{\mathrm{p}}$ & $0.69_{-0.11}^{+0.07}$ & $\rho_{\mathrm{J}}$ \\
Planet density, $\rho_{\mathrm{p}}(\mathrm{cgs})$ & $0.92_{-0.15}^{+0.09}$ & \\
Planet $T_{\text {eql }}(A=0 ; f=1)$ & $960 \pm 70$ & $\mathrm{~K}$ \\
\hline
\end{tabular}

Table 5. Parameters of the host star derived from the SME analysis of the FIES spectra and the MCMC analysis of the photometric and radialvelocity measurements.

\begin{tabular}{lcc}
\hline \hline Parameter & Value & Unit \\
\hline$T_{\text {eff }}$ & $4800 \pm 100$ & $\mathrm{~K}$ \\
$\log g$ & $4.45 \pm 0.2$ & \\
{$[M / H]$} & $0.0 \pm 0.2$ & \\
$v \sin i$ & $<6.0$ & $\mathrm{~km} \mathrm{~s}^{-1}$ \\
Stellar mass, $M_{\star}$ & $0.77_{-0.08}^{+0.10}$ & $M_{\odot}$ \\
Stellar radius, $R_{\star}$ & $0.74_{-0.03}^{+0.04}$ & $R_{\odot}$ \\
Stellar luminosity, $L_{\star}$ & $(1.00 \pm 0.11) \times 10^{33}$ & $\mathrm{erg} \mathrm{cm}^{-2} \mathrm{~s}^{-1}$ \\
\hline
\end{tabular}

\section{Discussion}

The system parameters derived here place WASP-11b towards the lower end of the mass range of known transiting planets, falling approximately mid-way between the masses of Jupiter and Saturn. The host star WASP-11 is also amongst the smallest and lowest luminosity stars known to host a transiting planet, however it is quite bright $(V=11.6)$. WASP-11b is irradiated by a stellar flux $F_{\mathrm{p}}=1.9 \times 10^{8} \mathrm{erg} \mathrm{cm}^{-2} \mathrm{~s}^{-1}$ at the sub-stellar point making it the third least heavily irradiated transiting planet after GJ436b and HD 17156b. We compute an equilibrium temperature for WASP-11b of $T_{\text {eql }}(A=0 ; f=1)=960 \pm 70 \mathrm{~K}$, which 

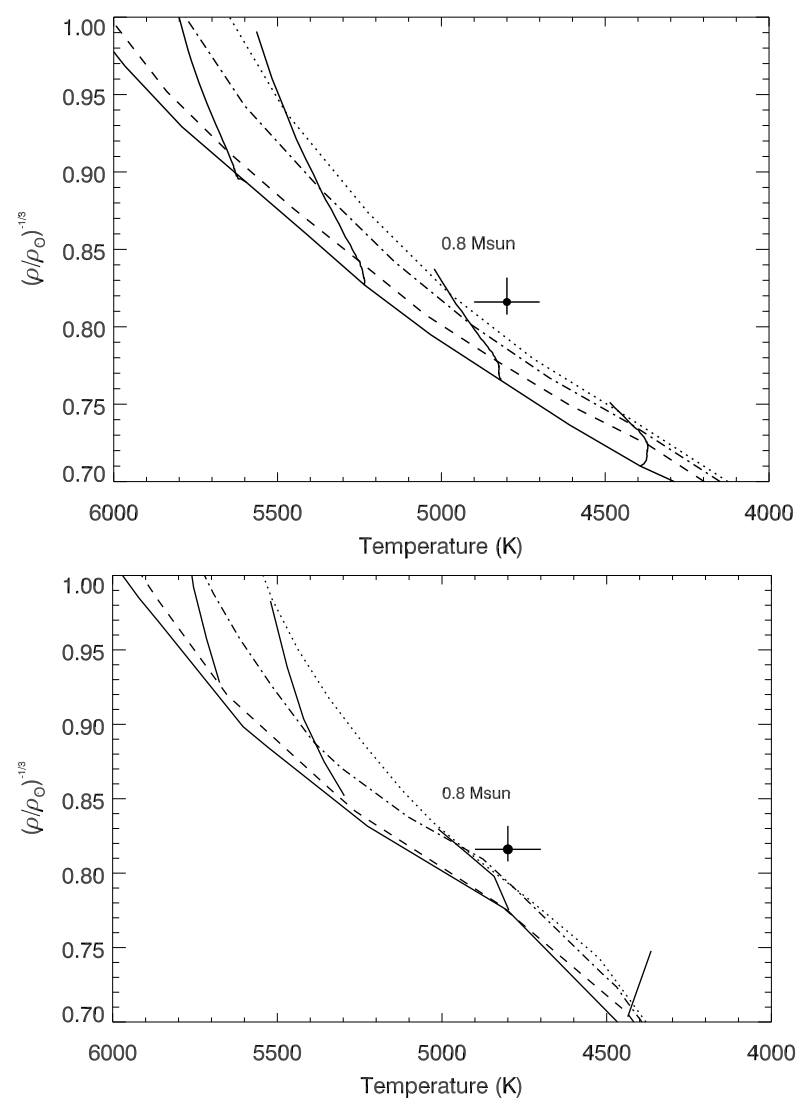

Fig. 5. The position of WASP-11 in the $R / M^{1 / 3}-T_{\text {eff }}$ plane. Evolutionary tracks for a solar metallicity star from Baraffe et al. (1998) (upper panel) and Girardi et al. (2000) (lower panel) are plotted along with isochrones for ages $10 \mathrm{Myr}$ (solid), $1 \mathrm{Gyr}$ (dashed), $5 \mathrm{Gyr}$ (dotdashed), $10 \mathrm{Gyr}$ (dotted). Evolutionary mass tracks are shown for 0.7, $0.8,0.9$ and $1.0 M_{\odot}$.

makes it more typical of the bulk of known exoplanets than of the "hot Jupiter" class most commonly found by the transit method.

Theoretical models of the atmospheres of hot giant exoplanets (Fortney et al. 2006; Burrows et al. 2007) have shown that heavy irradiation can lead to the development of a temperature inversion and a hot stratosphere. This is due to the absorption of stellar flux by an atmospheric absorber, possibly $\mathrm{TiO}$ and VO. In both sets of models the magnitude of the incident stellar flux is the key controlling variable determining whether a given extra-solar giant planet (EGP) will possess a hot stratosphere. Recent observations by Machalek et al. (2008) of secondary transits of XO-1b using the Spitzer Space Telescope suggest the presence of a temperature inversion in the atmosphere of that exoplanet. On the other hand analogous observations of HD 189733b (Charbonneau et al. 2008) show no evidence for an inversion, despite the irradiating fluxes of XO- $1 \mathrm{~b}$ and HD189733b being almost identical $\left(F_{\mathrm{p}}=0.49 \times 10^{9}\right.$ and $F_{\mathrm{p}}=0.47 \times 10^{9} \mathrm{erg} \mathrm{cm}^{-2} \mathrm{~s}^{-1}$ respectively). This strongly suggests that the incident stellar flux is not the sole controlling parameter determining the presence of the inversion, a likelihood which the authors of the atmosphere models readily point out themselves. Further observations of planets particularly in the low-irradiation regime are required to help parameterise the thermal inversion. WASP-11b is amongst the nearest and brightest low-irradiation EGPs making it a good candidate for such studies. Moreover we note that the currently available data suggest that the orbital eccentricity of WASP-11b is likely much lower
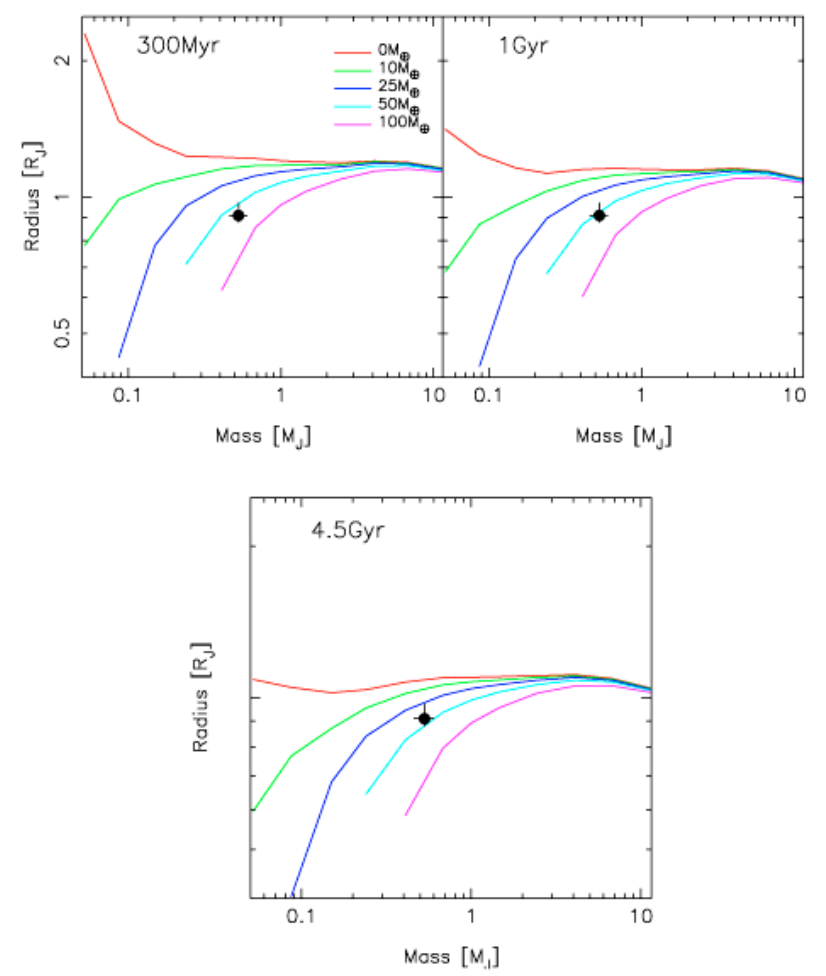

Fig. 6. Planetary mass-radius relations as a function of core mass and system age, interpolated from the models of Fortney et al. (2007).

than the other two bright low-irradiation transiting exoplanets, GJ436b and HD $17156 \mathrm{~b}$ ( $e=0.15$ and $e=0.67$ respectively). As a consequence the secular variation in irradiation around the orbit will be correspondingly lower in WASP-11b, removing a potentially complicating factor when comparing follow-up observations with predictions from atmospheric models developed assuming steady-state irradiation.

To estimate the age of the WASP-11 we compared the observed stellar density and temperature against the evolutionary models of low- and intermediate-mass stars of Girardi et al. (2000) and Baraffe et al. (1998). In Fig. 5 we plot the position of WASP-11 in the $R / M^{1 / 3}$ versus $T_{\text {eff }}$ plane atop isochrones of different ages from the two models. For such a cool star, the isochrones are closely spaced in this parameter plane due to the slow post-main-sequence evolution of late-type stars. The sets of isochrones from the two models overlap in this regime, and both models suggest the same mass and age for the host star. WASP-11 falls above the $10 \mathrm{Gyr}$ isochrone for both models, though it is consistent with this age within the errors. The very low lithium abundance also points toward WASP-11 being $\geq 1-2$ Gyr old (Sestito \& Randich 2005). We investigated using gyrochronology to age the host star, following Barnes (2007), however we were unable to measure a definite rotational period. No rotation modulation was detected in the lightcurve to an amplitude limit of a few milli-magnitudes. The spectral analysis furnishes only an upper-limit to $v \sin i$, so no rotational period can be determined in that way. Taken together these factors are all consistent with WASP-11 being an old star, older than maybe $1 \mathrm{Gyr}$, however it is not possible to be more definite than that with the available data.

Fortney et al. (2007) present models of the evolution of planetary radius over a range of planetary masses and orbital distances, and under the assumption of the presence of a dense core of various masses up to $100 M_{\oplus}$. To compare our results with the Fortney et al. models we plotted the modelled mass-radius 
relation as a function of core mass in Fig. 6. To account for the lower-than-Solar luminosity of the host star WASP-11 we calculated the orbital distance $a_{\odot}=a\left(M_{\star} / M_{\odot}\right)^{-3.5 / 2}$ at which a planet in orbit about the Sun would receive the same incident stellar flux as WASP-11b does from its host. We then interpolated the models of Fortney et al. to this effective orbital distance $\left(a_{\odot}=0.068\right.$ for WASP-11b). As the age of the WASP-11 system is poorly constrained we compared our results with the modelled mass-radius relation at $300 \mathrm{Myr}, 1 \mathrm{Gyr}$ and $4.5 \mathrm{Gyr}$. We find that the radius of WASP-11b is consistent with the presence of a dense core with a mass in the range $M_{\text {core }} \sim 42-77 M_{\oplus}$ for a system age of $300 \mathrm{Myr}, M_{\text {core }} \sim 33-67 M_{\oplus}$ at $1 \mathrm{Gyr}$, and $M_{\text {core }} \sim 22-56 M_{\oplus}$ at $4.5 \mathrm{Gyr}$. Clearly there is much uncertainty in the estimate of the core mass of WASP-11b due to the poor constraint on the age of the system. The core mass estimates are also inherently model-dependent, and differing estimates would result if additional physical processes were assumed to influence the radius of the planet.

Acknowledgements. The WASP Consortium consists of astronomers primarily from the Queen's University Belfast, Keele, Leicester, The Open University, and St Andrews, the Isaac Newton Group (La Palma), the Instituto de Astrofísica de Canarias (Tenerife) and the South African Astronomical Observatory. The SuperWASP-N and WASP-S Cameras were constructed and operated with funds made available from Consortium Universities and the UK's Science and Technology Facilities Council. SOPHIE observations have been funded by the Optical Infrared Coordination network (OPTICON), a major international collaboration supported by the Research Infrastructures Programme of the European Commission's Sixth Framework Programme. FIES observations were made with the Nordic Optical Telescope, operated on the island of La Palma jointly by Denmark, Finland, Iceland, Norway, and Sweden, in the Spanish Observatorio del Roque de los Muchachos of the Instituto de Astrofisica de Canarias. We extend our thanks to the Director and staff of the Isaac Newton Group of Telescopes for their support of SuperWASP-N operations, and the Director and staff of the Observatoire de
Haute-Provence for their support of the SOPHIE spectrograph. The Liverpool Telescope is operated on the island of La Palma by Liverpool John Moores University in the Spanish Observatorio del Roque de los Muchachos of the Instituto de Astrofisica de Canarias with financial support from the UK Science and Technology Facilities Council.

\section{References}

Bakos, G., Noyes, R. W., Kovács, G., et al. 2004, PASP, 116, 266

Baraffe, I., Chabrier, G., Allard, F., \& Hauschildt, P. H. 1998, A\&A, 337, 403

Barnes, S. A. 2007, ApJ, 669, 1167

Bouchy, F., \& The Sophie Team. 2006, in Tenth Anniversary of 51 Peg-b: Status of and prospects for hot Jupiter studies, ed. L. Arnold, F. Bouchy, \& C. Moutou, 319

Burrows, A., Hubeny, I., Budaj, J., Knutson, H. A., \& Charbonneau, D. 2007, ApJ, 668, L171

Charbonneau, D., Brown, T. M., Latham, D. W., \& Mayor, M. 2000, ApJ, 529, L45

Charbonneau, D., Knutson, H. A., Barman, T., et al. 2008, ArXiv e-prints, 802 Claret, A. 2000, A\&A, 363, 1081

Collier Cameron, A., Pollacco, D., Street, R. A., et al. 2006, MNRAS, 373, 799

Collier Cameron, A., Wilson, D. M., West, R. G., et al. 2007, MNRAS, 380, 1230

Fortney, J. J., Saumon, D., Marley, M. S., Lodders, K., \& Freedman, R. S. 2006, ApJ, 642, 495

Fortney, J. J., Marley, M. S., \& Barnes, J. W. 2007, ApJ, 659, 1661

Girardi, L., Bressan, A., Bertelli, G., \& Chiosi, C. 2000, A\&AS, 141, 371

Henry, G. W., Marcy, G. W., Butler, R. P., \& Vogt, S. S. 2000, ApJ, 529, L41

Machalek, P., McCullough, P. R., Burke, C. J., et al. 2008, ArXiv e-prints, 805

McCullough, P. R., Stys, J. E., Valenti, J. A., et al. 2005, PASP, 117, 783

O’Donovan, F. T., Charbonneau, D., Mandushev, G., et al. 2006, ApJ, 651, L61

Pollacco, D. L., Skillen, I., Cameron, A. C., et al. 2006, PASP, 118, 1407

Pollacco, D., Skillen, I., Collier Cameron, A., et al. 2008, MNRAS, 385, 1576

Queloz, D., Henry, G. W., Sivan, J. P., et al. 2001, A\&A, 379, 279

Sestito, P., \& Randich, S. 2005, A\&A, 442, 615

Stempels, H. C., Collier Cameron, A., Hebb, L., Smalley, B., \& Frandsen, S. 2007, MNRAS, 379, 773

Udalski, A., Paczynski, B., Zebrun, K., et al. 2002, Acta Astron., 52, 1 